\title{
Tratamiento informativo de candidatas presidenciales: una propuesta desde el framing
}

\section{News coverage on female presidential candidates: a proposal from framing}

Recibido: 28/06/2019

Edrei Álvarez-Monsiváis Aceptado: 03/10/2019 a00807373@itesm.mx

Publicado: 05/12/2019 Tecnológico de Monterrey (México)

Resumen: El artículo presenta una revisión de la literatura de las formas de abordaje periodístico hacia las candidatas presidenciales. Para este objetivo, se englobaron en siete encuadres de cobertura: $a$ ) visibilidad, b) reiteración del género, $c$ ) mediatización de la esfera íntima, $d$ ) personalidad, $e$ ) agenda temática, $f$ ) tonalidad y $g$ ) particularidades del medio. Finalmente, se formula una propuesta de operacionalización para cada uno de los encuadres que dé pie a futuras investigaciones que aborden a estas figuras políticas. Se concluye que estas son representadas desde su esfera íntima y feminidad, características que distan de ser las culturalmente apropiadas para el debate en la arena pública y política.

Palabras clave: Encuadres noticiosos, Candidatas presidenciales, Comunicación política, Género, Género y política, Tratamiento periodístico, Visibilidad, Campañas políticas.

Abstract: The paper presents a review of the literature of news coverage on female presidential candidates. For this purpose, seven coverage frames are included: a) visibility, b) gender reiteration, c) mediation of the intimate 
sphere, personality, d) thematic agenda, e) tonality and f) particularities of the media. Finally, an operationalization proposal is formulated for each of the frames that could be used in future research that addresses these political figures. It is concluded that women are represented from their intimate sphere and femininity, characteristics that are far from being culturally appropriate for debate in the public and political arena.

Key words: News frames, Female presidential candidates, Political communication, Gender, Gender and politics, News coverage, Visibility, Political campaigns.

\section{Introducción}

El presente trabajo tiene el objetivo de realizar una exploración teórica de las investigaciones que se han elaborado en torno al papel mediático de candidatas presidenciales. La intención es ofrecer categorías de análisis con indicadores de operación para estudios posteriores que aborden el tratamiento periodístico hacia aspirantes políticas.

El trabajo se justifica en la reciente participación de mujeres que han logrado consolidar una candidatura presidencial. Desde inicios del 2000 en América Latina, cuatro mujeres han ganado las presidenciales de su país en siete procesos electorales diferentes: Michelle Bachelet en Chile (elecciones de 2005 y 2013), Cristina Fernández en Argentina (2007, 2011), Laura Chinchilla en Costa Rica (2010) y Dilma Rousseff en Brasil (2010, 2014). En ese sentido, los medios de comunicación son imprescindibles para dar a conocer sus plataformas en tanto que se convierten en instrumentos importantes para la democracia de los países (Sánchez Ruiz, 2005).

Por medio del modelo del encuadre noticioso o framing, se englobaron en siete las formas de cobertura o frames desde los cuales se representa a las mujeres presidenciables. De esta forma, los encuadres de visibilidad, mediatización de la esfera íntima, reiteración del género, personalidad, agenda temática, tonalidad o valencia y particularidades del medio juegan como formas distintas de cubrir a mujeres que a hombres en política. 
El estudio contempla un escenario actual de las candidaturas de mujeres para el puesto ejecutivo en 19 países latinoamericanos, lo cual permite ahondar en la importancia de las investigaciones sobre estas figuras políticas. Posteriormente, se explica de qué manera se realiza el acercamiento de la propuesta hacia el modelo del framing. Después, se explora el estado de la cuestión de cada uno de los siete encuadres para análisis y, a partir de ahí, se presenta una propuesta de operacionalización que es posible adaptar para diferentes metodologías en comunicación política.

\section{Escenario actual de las candidatas presidenciales en Latinoamérica}

Aunque el modelo teórico-metodológico que está por esbozarse se configuró para operacionalizarse en distintos contextos, la región latinoamericana permite dar una perspectiva de cómo se encuentra el estado actual de las candidatas políticas. Entre las ventajas que tiene el analizar este escenario (y no otros) está que se tratan de países presidencialistas, con una larga tradición de pluralidad de partidos políticos, además de que el cargo se elige por voto directo de la ciudadanía.

El fijar la atención sobre la situación de las mujeres en política de alto rango en Latinoamérica permite vislumbrar la importancia que tiene la visibilidad y representación de los medios a estas candidaturas. En la tabla 1 es posible observar que 703 candidatas y candidatos han sido propuestos para el cargo presidencial del año 2000 al 2018 en 19 países de la región. De ese total, 76 pertenecen a candidaturas de mujeres, esto se traduce en apenas la décima parte (11\%). Además, existen tres países que durante ese tiempo no han propuesto mujeres para el Ejecutivo (El Salvador, Nicaragua y Uruguay). El resto (a excepción de Argentina y Perú) ha presentado, al menos, una elección en la que no participan aspirantes femeninas. 
Tabla 1: Presencia de candidatas presidenciales en América Latina ${ }^{1}$ (2000-2018).

\begin{tabular}{|c|c|c|c|c|c|c|c|}
\hline \multirow{2}{*}{ País } & \multirow{2}{*}{ Periodo } & \multirow{2}{*}{$\begin{array}{c}\text { Número } \\
\text { de } \\
\text { elecciones }\end{array}$} & \multirow{2}{*}{$\begin{array}{c}\text { Elecciones } \\
\text { con } \\
\text { candidatas }\end{array}$} & \multicolumn{2}{|c|}{ Candidatos } & \multicolumn{2}{|c|}{ Candidatas } \\
\hline & & & & $f$ & $\%$ & $f$ & $\%$ \\
\hline Chile & $2005-2017$ & 4 & 3 & 19 & 76 & 6 & 24 \\
\hline Venezuela & $2000-2018$ & 5 & 3 & 25 & 76 & 8 & 24 \\
\hline Argentina & 2003-2015 & 4 & 4 & 35 & 81 & 8 & 19 \\
\hline Brasil & 2002-2018 & 5 & 4 & 38 & 83 & 8 & 17 \\
\hline Perú & $2001-2016$ & 4 & 4 & 40 & 83 & 8 & 16 \\
\hline Colombia & $2002-2018$ & 5 & 4 & 33 & 85 & 6 & 15 \\
\hline México & $2000-2018$ & 4 & 3 & 17 & 85 & 3 & 15 \\
\hline Guatemala & $2003-2015$ & 4 & 3 & 43 & 88 & 6 & 12 \\
\hline Ecuador & 2002-2017 & 5 & 4 & 43 & 90 & 5 & 10 \\
\hline Paraguay & 2003-2018 & 4 & 3 & 34 & 92 & 3 & 8 \\
\hline Panamá & 2004-2014 & 3 & 1 & 13 & 93 & 1 & 7 \\
\hline $\begin{array}{l}\text { Rep. } \\
\text { Dominicana }\end{array}$ & $2000-2016$ & 5 & 1 & 25 & 93 & 2 & 7 \\
\hline Haití & $2000-2016$ & 4 & 3 & 92 & 94 & 6 & 6 \\
\hline Honduras & 2001-2017 & 5 & 2 & 30 & 94 & 2 & 6 \\
\hline Costa Rica & $2002-2018$ & 5 & 2 & 59 & 95 & 3 & 5 \\
\hline Bolivia & $2002-2014$ & 4 & 1 & 31 & 97 & 1 & 3 \\
\hline El Salvador & 2004-2014 & 3 & 0 & 11 & 100 & 0 & 0 \\
\hline Nicaragua & $2001-2016$ & 4 & 0 & 19 & 100 & 0 & 0 \\
\hline Uruguay & 2004-2014 & 3 & 0 & 20 & 100 & 0 & 0 \\
\hline \multicolumn{2}{|c|}{ Totales } & $\begin{array}{c}(n=80) \\
100 \%\end{array}$ & $\begin{array}{c}(n=45) \\
56 \%\end{array}$ & $(n=627)$ & $89 \%$ & $(n=76)$ & $11 \%$ \\
\hline
\end{tabular}

Fuente: Elaboración propia con datos oficiales de los países mencionados.

Además de esta baja proporción en las listas nominales de los partidos para competir por el puesto presidencial, es posible inferir que el electorado no ha beneficiado a las mujeres políticas. En la tabla 2, se encuentra que, de las 76 candidaturas de féminas, dos terceras partes (65\%) no ha alcanzado el $10 \%$ de votación, lo cual quiere decir que se trató de candidaturas no funcionales y $\sin$ impacto electoral.

1. Incluyen 19 países independientes con voto directo para las presidenciales. 
Una quinta parte del total de candidatas (20\%) obtuvo entre el 10 y $30 \%$ del total. Se tratan de candidaturas de mujeres populares que, si bien no era factible su triunfo, lograron atraer el voto para sus partidos y sentar precedentes de candidaturas de mujeres. Con más de $30 \%$ de votación, se encuentran 11 candidaturas de mujeres, donde se incluyen los siete periodos de mujeres presidentas, dos más que pasaron a la segunda vuelta y dos nominadas por el partido en el gobierno.

Tabla 2: Votación obtenida por las candidatas presidenciales.

\begin{tabular}{|c|c|c|}
\hline Voto obtenido & Frecuencia & Porcentaje \\
\hline Menos de $1 \%$ & 28 & 37 \\
\hline De 1 a $10 \%$ & 21 & 28 \\
\hline De 10 a $20 \%$ & 7 & 9 \\
\hline De 20 a $30 \%$ & 9 & 12 \\
\hline Más de $30 \%$ & 11 & $14 \%$ \\
\hline Total & $(n=76)$ & $100 \%$ \\
\hline
\end{tabular}

Fuente: Elaboración propia.

Con este escenario, es posible advertir la baja participación de candidatas en elecciones de alto rango y su poca penetración en el electorado. Algunos trabajos advierten que esto depende de factores estructurales como lo expresaría el índice de desarrollo humano (Araujo \& Alves, 2007); factores culturales, que tienen que ver con la posición de las mujeres en la sociedad (Valcárcel, 2008); y factores institucionales relacionados con los mecanismos de los partidos para candidatear mujeres (Costa, Bolognesi \& Codato, 2013).

En este trabajo, se plantean los factores de representación mediática. La importancia recae en que la forma en la evidencien las candidaturas pueden llegar a tener un impacto en el electorado. Llanos (2014) y Rodelo (2016) coinciden en que, a pesar de que las candidaturas de mujeres son menores en proporción a la de los hombres, la cantidad de información que los medios generan sobre ellas es aún menor, lo cual pone en jaque la efectividad de las leyes de cuota de género que son un estandarte en las leyes de partido en la región. 


\section{Modelo del encuadre noticioso}

Para términos de la presente propuesta, se ha decidido nombrar a las categorías de análisis bajo el concepto de "encuadres mediáticos". El acercamiento conceptual se hace necesario debido a que denominarlas "encuadres" permite brindarles los presupuestos que el framing persigue.

Bajo lo que se denomina como el "segundo nivel de la agenda", el framing se ha propuesto estudiar cómo, mediante la repetición de atributos dentro del mensaje informativo, los medios generan información sobre qué pensar de un tema. Entman (1993) explicó que el encuadre se trata de la selección de aspectos de una realidad percibida para producir un mensaje con el propósito de promover una definición y evaluación particulares. En ese sentido, los encuadres transmiten diferentes formas de ver la realidad (Aruguete, 2011).

De esta manera, la propuesta del encuadre noticioso no se enfoca solamente en la relevancia que los medios le otorgan a un tema sobre otro, sino en la forma de abordaje, ya que se buscan causas e interpretaciones sobre el problema mediante la selección, énfasis y exclusión de información (Semetko \& Valkenburg, 2000; Tankard, 2001)

Con base en estas definiciones, la propuesta para revisar el tratamiento mediático a candidatas presidenciales se basa en el establecimiento de frames, debido a que su medición, en términos de reiteración o desestimación de los componentes que configuran cada uno de los encuadres, permitirá reconocer cuáles son las definiciones, interpretaciones y evaluaciones que los medios transmiten en sus mensajes periodísticos. De esta forma, cada uno de los siete encuadres que a continuación se presentan, tienen el fin de desmembrar los componentes de un mensaje mediático, con el objetivo de encontrar cuáles son esos elementos que configuran la representación de candidatas.

El acercamiento a este modelo no se considera que afecte la metodología desde la cual se aproximen las investigaciones que de ella deriven ya que el modelo parte desde dos aproximaciones distintas. Por un lado, en la perspectiva directa se encuentra la predisposición de palabras o elementos clave, con el fin de medir su cantidad o ausencia dentro del texto periodístico (Tankard, 2001). Desde la perspectiva dinámica, Gamson \& Modigliani (1989) explican que se trata de la interpretación de signos a través de textos con carga simbó- 
lica los cuales pueden ser metáforas, ejemplos, eslóganes, representaciones o imágenes. Es interesante ver estas distinciones entre las perspectivas a las que hacen referencia López \& Vicente (2013), en tanto que la directa parece ser más funcionalista, mientras que la dinámica mantiene un análisis crítico-discursivo.

De esta manera, la propuesta que a continuación se presenta se define como un modelo de siete encuadres que proponen su exploración mediante la medición y el análisis interpretativo de los marcos noticiosos, en orden de revelar un tratamiento específico para candidatas presidenciales.

\section{Encuadres noticiosos en las candidaturas de mujeres en política}

\subsection{Encuadre 1: visibilidad}

La visibilidad es un elemento importante para la representación de personajes en los medios. De acuerdo con Gerbner \& Gross (1976), si la "representación en el mundo ficticio significa existencia social; la ausencia significa aniquilación simbólica" (p. 45). En ese sentido, el no generar información sobre mujeres candidatas complica la existencia en la conciencia del electorado y que, por ende, no apoyen su proyecto (Everitt, 2003).

Por tanto, se hace necesario revelar qué tan visibles son las mujeres políticas en los medios. La propuesta de análisis de tratamiento en visibilidad contesta a tres preguntas: "cuánto", "en dónde" y "con quién" aparecen las candidatas. En primera instancia, se tiene una discrepancia entre investigaciones sobre la cantidad de información que ha surgido sobre mujeres aspirantes. Los análisis de contenido en la prensa estadounidense durante los años noventa arrojaron que el número de párrafos dedicados a postulantes al Senado era mayor cuando se trataba de hombres (Kahn \& Goldenberg, 1991; Kahn, 1992, 1994). El motivo parecía ser que las mujeres aún no obtenían escaños suficientes, por lo que la probabilidad de que ganaran era mínima y poco noticiosa.

Sin embargo, existen trabajos después de los años 2000 en primarias presidenciales (Heldman, Carroll \& Olson, 2005), campañas a la gubernatura (Bryant, 2014) y elecciones parlamentarias (Hooghe, Jacobs \& Claes, 2015), donde la cantidad de información sobre candidatas fue hasta tres veces menor que la de los hombres. 
Por otra parte, se encontraron investigaciones que demuestran que las mujeres han obtenido hasta el doble de cobertura que los hombres (Aday \& Devitt, 2001; Bystrom, Robertson \& Banwart, 2001; Banwart, Bystrom \& Robertson, 2003; Jalalzai, 2006; Wasburn \& Wasburn, 2011; Miller \& Peake, 2013; Bertrand, 2016). La explicación de una cobertura importante hacia las candidatas puede ser fruto de la novedad que supone el ver a una mujer como aspirante a un cargo político mayor (Fernández García \& Tous Rovirosa, 2012).

Otros estudios más indican que en diferentes contextos se ha logrado la igualdad en la cobertura. Tales son los casos de las elecciones alemanas de 2005 (Semetko \& Boomgaarden, 2007) y las primarias presidenciales de 2008 en Estados Unidos (Fernández García, 2010). De acuerdo con estos trabajos, la equidad de cobertura se ha mantenido estable en los últimos 20 años en países anglosajones (Kittilson \& Fridkin, 2008), así como en Holanda (Bijker, 2015) y Canadá (Wagner, Trimble, Sampert \& Gerrits, 2017).

A partir de las discrepancias entre estos tres bloques de estudios, se puede inferir que la cantidad de visibilidad no depende de si se trata de un candidato o candidata, sino más bien de contextos y personajes específicos. Por tanto, es deseable continuar con trabajos que expongan el volumen de cobertura que reciben las políticas.

Por el lado del "dónde", se ubican las candidatas en el mensaje periodístico, se han encontrado tres distintos espacios para abordarlas. Un primer espacio es el referente al lenguaje mediático. Los autores Wasburn \& Wasburn (2011) indicaron que las mujeres reciben mayor atención mediática a través de imágenes; mientras que los hombres, en texto. De esta manera, los trabajos que analizan el contenido de medios audiovisuales encontraron mayor cantidad de información hacia candidatas que para candidatos, como es el caso de las elecciones mexicanas de 2012 en los telediarios (Martínez \& Godínez, 2013). El estudio sobre noticias en línea de Humprecht \& Esser (2017) coincide en que existe mayor lenguaje visual para abordar a las aspirantes. En ese sentido, los medios darían mayor importancia a la apariencia en cuanto a iconografía para las mujeres, y mayor profundidad temática en cuanto a texto para los hombres.

Un segundo espacio es el encontrado por Bryant (2014), quien indica que la información sobre mujeres en prensa se encuentra en mayor medida en edi- 
toriales; mientras que las de los hombres, en las notas informativas. De esta manera, la oportunidad de recibir mayor cantidad de hechos noticiosos asépticos sería para los hombres, mientras que los datos sobre las mujeres estarían ya filtrados por un analista del medio. Lo anterior daría lugar a menor oportunidad al electorado de hacerse una perspectiva propia sobre las plataformas políticas de mujeres.

Un tercer espacio es a través de la forma de citar a las candidatas. Humprecht \& Esser (2017) encontraron que los hombres obtienen mayor número de citas directas en las notas. De esta forma, los candidatos tendrían mayor oportunidad de fijar sus posturas bajo sus propias palabras, mientras las opiniones de las aspirantes se encontrarían parafraseadas o anuladas.

El último aspecto por reparar en cuanto a visibilidad es quiénes son las personas que más aparecen junto a las candidatas en el mensaje informativo. Ríos Sierra (2017) concluyó que las notas periodísticas remiten a otras personas (principalmente hombres) para recordar que la campaña se apoya o no hubiera sido posible sin la ayuda de alguien más. A este comportamiento lo nombra "corresponsabilidad", en tanto los medios sustentan la importancia política de las mujeres en personajes como su esposo (Cristina Fernández con Néstor Kirchner), padre (Keiko Fujimori con Alberto) o el presidente de turno (Dilma Rousseff, "la candidata de Lula").

Para Anderson \& Sheeler (2005), definir a las políticas a partir de su relación con hombres influyentes, trivializa sus méritos y levanta sospechas sobre la legitimidad de sus logros, mientras que son encuadradas como manipulables para los intereses de alguien más. Ante esto, Ehrlich \& King (1992) ya habían enfatizado en el hecho de conocer a las mujeres políticas en sus propios términos más que en su relación con otros. Así, la presencia de familia, políticos, partidarios, empresarios entre otros personajes puede participar en restarle visibilidad a los logros individuales de las mujeres.

\subsection{Encuadre 2: mediatización de la esfera íntima}

La esfera íntima fue conceptualizada por Habermas (1989) como aquella en la que se tocan temáticas domésticas, familiares y corpóreas. Asimismo, Arendt (2009) enfatizó que lo íntimo no tiene interés público ni es de importancia 
para el bien común. Sin embargo, por una cuestión cultural donde las mujeres son las fieles representantes de esta esfera, al momento de buscar integrarse a una esfera pública y política, traen impregnadas estas cuestiones que llaman la atención de los medios (De Fontcuberta, 1990).

Son dos los rasgos generales que se han encontrado en la mediatización de la esfera íntima. En primer lugar, los medios parecen estar interesados en conocer el estatus marital y familiar de las aspirantes (Bystrom, Robertson \& Banwart, 2001; Falk, 2010). De acuerdo con García Beaudoux (2014), en la televisión, se conoce el estado civil de más del 50\% de las mujeres que aparecen en pantalla, contra menos del 30\%, en el caso de los hombres. Lo cual es consistente con lo encontrado por Fernández García (2010), quien describió que, durante las primarias demócratas de 2008, se citó el estado civil o al cónyuge de Hillary en el $46 \%$ de los casos, mientras que para Obama el porcentaje era apenas del $4 \%$ de las notas.

Las investigaciones también señalan que los medios suelen reparar en quiénes son sus hijos, hermanos y padres (Bystrom, Robertson \& Banwart, 2001; Falk, 2010; García Beaudoux, D’Adamo \& Gavensky, 2018). La noticiabilidad de estos datos recae en la idea cultural de que conocer de qué familia viene y qué tan buena madre y esposa ha sido, permitirá saber si puede replicar su labor de madre, ahora, de la nación (Martínez-Lirola, 2010).

El segundo tema dentro de la esfera íntima es la apariencia y estilo de las candidatas. Existe una variedad de casos donde, mientras las mujeres políticas fijan postura sobre un tema importante, la noticia es la manera en la que se arregló para el evento. Por ejemplo, Stein (2009) narra cómo, en 2007, Clinton dio un discurso en el Senado sobre el alto costo de la educación, mientras que la nota con mayor repercusión fue una crítica del Washington Post acerca de su escote que se encontraba bajo un saco rosa.

Otras investigaciones han analizado cómo los medios invitan a su audiencia a fijar su atención en la apariencia física de las mujeres políticas en diferentes contextos (Adams, 2011; Wasburn \& Wasburn, 2011; White, 2012; Agostinho Martins, 2013; Dan \& Iorgoveanu, 2013; Miller \& Peake, 2013; Bijker, 2015; Conroy, Oliver, Breckenridge-Jackson \& Heldman, 2015; Rincón Soto, 2015). Fernández García \& Tous Rovirosa (2012) citan los ejemplos mediáticos sobre la boina de Cristina Fernández en París en 2008, el traje de la 
Ministra española Carme Chacón durante la pascua de 2009 y el vestido de Ángela Merkel en la Ópera de Oslo de 2008.

Sin embargo, la apariencia también puede ser más masculina que femenina, por lo que los medios, además de fijar su atención sobre su vestuario rupturista, le podrían dar una tonalidad negativa. Carlin \& Winfrey (2009) encontraron a una Sarah Palin retratada desde su sensualidad femenina, mientras que Hillary Clinton como insuficientemente femenina al usar pantalones. El hecho de que la apariencia y figura formen parte de "la discusión mediática muestra que la coexistencia de poder y feminidad aún no se acepta y provoca irritación en el discurso mediático" (Lünenborg \& Maier, 2014, p. 187).

En ese sentido, este encuadre debe estar dedicado a evidenciar en qué medida los medios se acercan más a evidenciar credenciales personales de las candidatas (mujer, madre, esposa, hija) que las credenciales políticas (puestos políticos, académicos y de liderazgo). Foster Shoaf \& Parsons (2016), clasifican en las categorías de "hair", "husband" y "hemline" (cabello, pareja sentimental y vestimenta), donde encontraron que el $65 \%$ de las noticias en televisión y prensa de Estados Unidos sobre candidatas políticas, tiene alguno de estos aspectos, lo cual fue de solo $35 \%$ para candidatos.

Además de revelar un carácter sexista de los medios, encuadrarlas desde su esfera íntima tiende a despolitizar y trivializar las labores de las candidatas (García Beaudoux, D’Adamo \& Gavensky, 2018), lo cual dificulta el paso de las mujeres hacia el ámbito político ya que el electorado no percibe su legitimidad política, sino su plano familiar. Ante esto, Van Acker (2003) afirmó que una política se encuentra en constante resistencia en convertirse en una celebridad más que en una contendiente, por lo que es relevante la investigación de estos criterios de abordaje.

\subsection{Encuadre 3: reiteración del género}

Para Clark (1992), "nombrar es una poderosa herramienta ideológica" (p. 209), en tanto que diferentes nombres generan diferentes formas de representación. En ese sentido, la investigación de Fernández García (2010) hacia las primarias demócratas de 2008 encontró tres "formas generizadas" de nombrar a las mujeres en política. Primero, a través de explicitar el género por medio 
de palabras que enfaticen que se trata de una mujer en busca de candidatura. Para Hillary Clinton existió un $27 \%$ de artículos que la mencionaron como mujer, lo cual no fue de manera similar con Barack Obama con solamente el $3 \%$ de los casos que lo referenciaron como hombre.

Heldman, Carroll \& Olson (2005) concuerdan con lo anterior al encontrar que el hecho de ser mujer en una carrera presidencial fue lo noticioso en los artículos de fondo de Elizabeth Dole en las primarias republicanas. Para Ditonto (2014), este comportamiento genera la idea de que se trata de una candidata para las mujeres y no de la totalidad del padrón electoral.

La segunda forma de reiterar el género es través de las maneras de nombrar a la candidata. Fernández García (2010) explica que los medios mencionan con mayor formalidad a los hombres que a las mujeres en políticas. De esta manera, se encontró la palabra Hillary sin su apellido en un $34 \%$ de los casos, mientras que Obama, el apellido del candidato, apareció hasta en el 74\% (Uscinski \& Goren, 2010). Se entiende como una reiteración del género, en tanto un apellido no tiene impregnado el género en sí mismo, mientras que los nombres sí son clasificados en femeninos y masculinos.

En investigaciones posteriores, Sensales, Areni \& Dal Secco, (2016) notaron que los hombres ministros italianos fueron abordados desde su apellido en el $75 \%$ de las notas, mientras que las ministras en el 50\%. Estas últimas también registraron mayor frecuencia en ser nombradas a partir de su nombre de pila. Mientras que el trabajo de Power (2017) indicó que las mujeres en puestos de liderazgo en Reino Unido reciben un cuarto de las noticias totales con su nombre de pila, mientras que un discreto $3 \%$ desde su apellido.

La investigación de Page (2003) confirma lo anterior y aporta, además, que la forma de nombrar a una mujer política, como fue el caso de Cherie Blair, dependerá del tratamiento específico: a) cuando fue nombrada por su nombre de pila, se trataba de noticias sobre celebridades y caritativas; $b$ ) se utilizó su apellido de casada cuando la nota la encuadraba desde su rol de madre y; c) su apellido de soltera apareció en información legal.

La última forma de reiterar el género es a través de la verba dicendi. Se tratan de verbos utilizados en los mensajes periodísticos que indican "que un acto lingüístico ha sido realizado" (Maldonado, 1999, p. 3559). Estos pueden ser 
divididos en dos tipos: los "neutrales" que indican el acto de decir, tales como mencionar, decir y firmar; y los "emocionales", que enuncian orden o mandato con una fuerza ilocutiva, entre los cuales se encuentran exclamar, preguntar y reclamar (Vicente Mateu, 2007).

En ese sentido, se encuentran dos vertientes diferentes de críticas hacia los verbos que escogen los medios para describir las acciones de las candidatas. Por un lado, Fernández García (2010) indica que estos suelen ser más neutrales hacia las candidatas, por lo que el periodista no enfatiza las acciones de la candidata, lo cual tiende a la despolitización. Por el otro, Gidengil \& Everitt (2003) aseguran que citar a las mujeres por medio de verbos "combativos" (como discutir, explotar, lanzar ataque, burlarse, rechazar y culpar) generan una representación de las candidatas como "inestables" o emocionales, lo cual no concuerda con lo esperado de una figura política.

Enfatizar o no (de forma emocional) las acciones de las políticas las sitúan en una frontera frágil entre concebir a las candidatas como "lideresas políticas" y como "ambiciosas por el poder", de acuerdo con Holtz-Bacha (2013). El reiterar que se trata de una mujer en campaña a través de explicitar la palabra "mujer", nombrarla desde su nombre de pila (en lugar desde su apellido) y utilizar verbos combativos para realzar la emotividad, son indicadores de que las mujeres son encuadradas primero por ser "mujeres" y después como "políticas" (Ross, 2002).

\subsection{Encuadre 4: personalidad}

De acuerdo con Kahn (1994), los medios de comunicación mantienen una fijación en el carácter de los políticos para evaluar fortalezas y debilidades. Sin embargo, se ha encontrado que, cuando se tratan campañas donde participan mujeres, la fijación hacia la personalidad aumenta (Heldman, Carroll \& Olson, 2005; Dunaway, Lawrence, Rose \& Weber, 2013; Allen, 2016). Para VanHorn (2010), esto ocurre porque los candidatos se ven obligados a mostrar su lado humano en una elección; sin embargo, mientras que los hombres lo hacen a través de sus sustitutos (como su esposa o familia) las mujeres lo hacen desde sí mismas, sin ayuda de otros, lo cual tiende a mostrarlas desde un enfoque sensacionalista y trivial (Trimble, Wagner, Sampert, Raphael \& Gerrits, 2013). 
Los antecedentes de investigación coinciden en que la arena política muestra dos tipos de personalidades de acuerdo con el género. La personalidad de femenina se acerca a los atributos de afabilidad, atracción, bajo perfil, carisma, cortesía, dependencia, emotividad, empatía, fama, honestidad, pacificidad, sensibilidad, volatilidad y vulnerabilidad (Kahn, 1994; Van Acker, 2003; Valenzuela \& Correa, 2006; Kittilson \& Fridkin, 2008; López-Hermida, 2009; Lemarier-Saulnier \& Lalancette, 2012; Lünenborg \& Maier, 2014). Dichos rasgos tienen que ver con el ser para otros y son aprendidos desde un espacio íntimo y privado.

Los atributos de agresividad, confianza, convencimiento, competitividad, comandancia, decisión, dureza, efectividad, estadista, experiencia, frialdad, fuerza, independencia, inteligencia, liderazgo, optimismo, pensador, rigor, trabajador y vital se engloban en una personalidad masculina (Kahn, 1994; Van Acker, 2003, Aalberg \& Jenssen, 2007: Kittilson \& Fridkin, 2008; Lemarier-Saulnier \& Lalancette, 2012; Lünenborg \& Maier, 2014), los cuales son aprendidos en la arena pública.

Es claro, entonces, que los rasgos masculinos son vistos como los más adecuados para una personalidad política. Aunque ciertos atributos femeninos (como la honestidad y empatía) podrían jugar positivamente en una candidatura (Franceschet, 2006), los medios han tenido que masculinizar las acciones de las mujeres para que ellas puedan ser competitivas en las elecciones. De esta manera, se encuentra una ocultación de la personalidad femenina de las mujeres en diferentes contextos (Kittilson \& Fridkin, 2008; Meeks, 2013), donde los hombres se encuentran posibilitados para utilizar su personalidad masculina.

La tendencia en la cobertura de candidatas políticas es hacia la masculinización de su personalidad; empero, también se halló que cuando la personalidad masculina se encuentra muy presente, las mujeres son catalogadas como frías, calculadoras y ambiciosas, lo que les merece una tonalidad negativa en los mensajes periodísticos (Harp, Loke \& Bachmann, 2010; Quevedo \& Suárez-Romero, 2017). En ese sentido, la estrategia parece ser una mezcla andrógina en la personalidad, como el que señalan Carlin \& Winfrey (2009) que se logró en la candidata republicana Sarah Palin.

Jamieson (1995) utiliza el concepto del "doble vínculo" (double bind) para referirse al doble mensaje que recibe la mujer en puestos de liderazgo. Por 
un lado, se le invita a seguir ciertos comportamientos que culturalmente debe mantener con respecto a su género. Por el otro y al buscar ingresar a un puesto de alto rango, se requiere que ella tenga una personalidad ajustada más hacia rasgos masculinos. Así, la estructura creada, la cual es resultado de costumbres anteriores (Hall, 1974), recae sobre la acción de la mujer política, quien se encuentra en una disyuntiva entre actuar de manera femenina o masculina para encajar en política.

\subsection{Encuadre 5: agenda temática}

Así como existen estudios que sustentan una separación de personalidad de acuerdo con el género, también existen trabajos que indican una agenda de temas para mujeres y otra para hombres. En la primera, se presentan tópicos que tienen que ver con tópicos de cuidado y los que afectan específicamente a las mujeres, como son aborto, arte, asistencialismo, ciencia, cuidado de niños, cultura, deportes, educación, empleo, energía, familia, justicia social, medio ambiente, migración, pobreza, salud, tercera edad, transporte, violencia contra las mujeres y derechos de minorías y reproductivos (Finamore \& Coin de Carvalho, 2006; Gómez-Escalonilla, García, Santín, Rodríguez \& Torregrosa, 2008; Morales, 2008; López-Hermida, 2009; Wasburn \& Wasburn, 2011; Fernández García \& Tous Rovirosa, 2012; Vos, 2013; Bertrand, 2016; Humprecht \& Esser, 2017).

En cambio, la agenda masculina está compuesta por temáticas como agricultura, corrupción, crimen, defensa, economía, elecciones, infraestructura, legalidad, negocios, política exterior, seguridad y terrorismo (Finamore \& Coin de Carvalho, 2006; García, Gómez-Escalonilla, Torregrosa, Rodríguez \& Santín, 2008; Gómez-Escalonilla, García, Santín, Rodríguez \& Torregrosa, 2008; Morales, 2008; Fernández García \& Tous Rovirosa, 2012; Vos, 2013; Humprecht \& Esser, 2017). Los tópicos mencionados hacen referencia a las labores de los hombres de ser protectores, proveedores y negociadores, que culturalmente les han atribuido.

Los temas que se le atañen históricamente a las mujeres pertenecen a las llamadas "carteras de segunda categoría", debido a que (aunque son muy importantes en una nación) se insertan más en un círculo con menor prominencia en la defensa de la soberanía y, por tanto, no suelen ser prominentes para 
la decisión del voto de la ciudadanía (Fernández Poncela, 2008). Además, durante las elecciones, dichas temáticas no alcanzan una cobertura suficiente por parte de los medios (Meeks, 2013; Vos, 2013; Martínez \& Maltos, 2019). De esta manera, las mujeres deben relegar tareas que culturalmente le son atribuidas, mientras que apropian otras asignadas a su sexo opuesto.

Para Atkeson \& Krebs (2008), lo anterior parece ser más una ventaja que desventaja, debido a que, por ser mujeres, se espera que asuman tópicos femeninos, mientras que, por ser políticas, se espera su involucramiento en temas de orden superior. Wasburn \& Wasburn (2011) hallaron que, aunque una candidata tenga consigo una agenda de tópicos importantes para la soberanía nacional, los medios prefieren preguntarle desde ámbitos asistencialistas, hacia los cuáles consideran que deberían poseer una opinión.

Para López-Hermida (2009) y Humprecht \& Esser (2017), el contexto del país influye en la agenda que se tendrá para cubrir a candidatas y candidatos. Por ejemplo, Monfardini (2013) describe que, aunque Dilma Rousseff fue cubierta desde temas sociales, esto le jugó a favor, debido a que la educación y la salud eran tareas urgentes por abordar en Brasil. En ese sentido, aunque la coyuntura del país definirá la agenda mediática, en lo que coinciden estos estudios es en que existe una clasificación de agendas de acuerdo con el género que, en la mayoría de los casos, la femenina resulta con menor cobertura e importancia para el electorado.

\subsection{Encuadre 6: tonalidad o valencia}

La tonalidad o valencia es utilizado en los estudios de comunicación política para evaluar temas o situaciones con el fin de conocer si estos fueron abordados de términos positivos o negativos (De Vreese \& Boomgaarden, 2003). Para Schuck \& De Vreese (2006) y Hooghe, Jacobs \& Claes (2015), la tonalidad del contenido informativo tiene un papel importante en las percepciones, juicios, evaluaciones y comportamiento de los actores políticos que se representan, por lo que es relevante reparar en ella en los trabajos de framing.

De acuerdo con los estudios sobre la tonalidad del mensaje periodístico sobre mujeres políticas, se ha encontrado que ellas suelen tener mayor valencia ne- 
gativa que sus contrincantes hombres. En los noventas, el tono desfavorable se hacía presente en la inviabilidad de las campañas de mujeres, donde se suponía que (por la baja presencia de políticas en cargos) estas se encontraban en desventaja (Kahn \& Goldenberg, 1991; Kahn, 1992).

Sin embargo, estudios recientes demuestran que esta la tonalidad negativa continúa presente en mayor medida hacia las mujeres (Fernández García, 2010; Miller \& Peake, 2013; Bryant, 2014; Bijker, 2015). Quevedo \& Suárez-Romero (2017) explican que la valencia dependerá de la personalidad desde las que se aborden a las candidatas. Cuando una mujer política se presentaba desde atributos masculinos, su imagen parecía severa y distante, por lo que la nota tornaba desfavorable. Por otro lado, si se ofrecían fotografías y texto que resaltaran su feminidad, la nota tendía a favorecer la imagen pública de la candidata (Dan \& Iorgoveanu, 2013).

Sobre el tipo de agenda temática se generó un comportamiento similar. Si las mujeres vierten opiniones en temáticas culturalmente establecidas para hombres (como lo son política exterior y economía), la valencia de la información era negativa (Wachai, 2005). Sobre la estrategia para establecer la tonalidad negativa y la neutral o positiva, Allen (2016) recomienda encasillar en esta información lo relacionado con corrupción y conflicto de interés. Mientras que Wachai (2005) exploró los verbos, adjetivos y adverbios utilizados para describir a las mujeres políticas.

\subsection{Encuadre 7: particularidades del medio}

Existen tres características de los medios que son importantes analizar al momento de politizar o despolitizar a las aspirantes. La primera de ellas es el sexo del reportero o comunicador. Para Aday \& Devitt (2001), cuando un hombre es el que firma las noticias en prensa, la candidata es tratada de manera menos politizada, es decir, en términos de ella como mujer y su esfera íntima, además que sus declaraciones eran parafraseadas; mientras que, cuando la autoría fue de una reportera, las candidatas tendían a ser encuadradas en temas políticos y su voz estuvo presente en citas directas. Whalen (2016) coincide con este estudio, al demostrar que los reporteros firman más notas donde se muestran las credenciales personales de las candidatas. 
En televisión se encontró el mismo fenómeno, puesto que Cassidy (2012) concluyó que los conductores hombres tendieron a presentar a las mujeres en roles tradicionales, mientras que las conductoras y grupos mixtos, las cubrían a través de temas masculinos y controversias políticas. En ese sentido, si al menos una mujer interviene en la creación de contenido, las candidatas suelen ser más politizadas.

Sin embargo, otra serie de estudios señala que las reporteras tienden a describir a las candidatas en términos de apariencia (Heldman, Carroll \& Olson, 2005). Vos (2013) coincide con esta perspectiva y explica que, aunque las reporteras son las que mayormente cubren a las mujeres, lo hacen desde noticias más humanas, mientras que los hombres a través de un corte más duro y politizado. Por tanto, si bien no parece haber un consenso sobre si son las comunicadoras o los comunicadores los que despolitizan en mayor medida a las candidatas, es importante continuar su exploración en orden de comprobar que no existe un sesgo en este aspecto. Lo anterior es aún más relevante si se toma en cuenta que la mayoría de las redacciones está compuesta por hombres (Vos, 2013).

La segunda particularidad de los medios en reparar es el espectro político. Cassidy (2012) comprobó la hipótesis que los canales de noticias conservadores tendieron a mostrar a las lideresas políticas en el marco de roles tradicionales de las mujeres, mientras que los liberales preferían no resaltar el género en tanto que les resultara natural el que una mujer ostente o persiga un cargo público. Además de ello, los medios conservadores concentraron la mayor cantidad de información con valencia negativa.

La última particularidad es que los medios que presentan información más audiovisual que textual, serán los que tiendan a la despolitización de las candidatas políticas. Así, los trabajos en televisión (Cassidy, 2012), tabloides (Dan \& Iorgoveanu, 2013) y revistas de alta gama (Berrocal \& Quevedo, 2014) tienden a trivializar, a través de sus imágenes, las funciones y posturas de las candidatas.

\section{Propuesta de operacionalización de encuadres}

En la tabla 3 se describen los siete encuadres que se han discutido anteriormente. Cada uno de estos tiene categorías o variables con sus propios indi- 
cadores. Como se mencionó desde un principio, el abordaje metodológico se puede realizar en términos tanto cuantitativos como cualitativos.

Desde cada uno de los encuadres se pueden obtener hallazgos específicos. En el encuadre de visibilidad se presentan los datos que tienen que ver con cantidad de información, elementos lingüísticos utilizados, lugares dentro del medio, presentación de la voz de la candidata y personajes que participan con ella en el mensaje. El objetivo de este encuadre es, entonces, conocer si existen desventajas en la cantidad y estructura noticiosa en la que son representadas las candidaturas de mujeres.

Tabla 3: Propuesta de operacionalización para el análisis del tratamiento mediático hacia candidatas presidenciales.

\begin{tabular}{|c|c|}
\hline Categoría & Indicadores \\
\hline \multicolumn{2}{|r|}{ Encuadre 1: Visibilidad } \\
\hline Cantidad & $\begin{array}{l}\text { Extensión del texto, tiempo aire, número de imágenes, infográficos } \\
\text { o videos }\end{array}$ \\
\hline Lenguaje & Texto, imágenes, sonido y videos \\
\hline Espacio & $\begin{array}{l}\text { Género opinativo o informativo; portada o interiores; encabezados } \\
\text { o cuerpo del mensaje; posición en la escaleta de programas tele- } \\
\text { visivos o radiofónicos }\end{array}$ \\
\hline Declaraciones & Citas directas o bites, paráfrasis o mensajes sin declaraciones \\
\hline $\begin{array}{l}\text { Personajes en la } \\
\text { información }\end{array}$ & $\begin{array}{l}\text { Pareja sentimental, familia, partidarios, empresarios, ciudadanos, } \\
\text { organizaciones, gabinete presidencial y celebridades }\end{array}$ \\
\hline \multicolumn{2}{|r|}{ Encuadre 2: Mediatización de esfera íntima } \\
\hline Apariencia y estilo & Vestimenta, peinado y movimientos (femenino o formal/masculino) \\
\hline $\begin{array}{l}\text { Credenciales } \\
\text { personales }\end{array}$ & $\begin{array}{l}\text { Mujer, señora, esposa, madre, hija, hermana, entre otras relacio- } \\
\text { nes con respecto a su familia extensiva }\end{array}$ \\
\hline $\begin{array}{l}\text { Credenciales } \\
\text { profesionales }\end{array}$ & $\begin{array}{l}\text { Títulos académicos, cargos políticos y profesionales, estatus de } \\
\text { candidata y direcciones de organizaciones }\end{array}$ \\
\hline \multicolumn{2}{|r|}{ Encuadre 3: Reiteración del género } \\
\hline Ser mujer & Palabras mujer o mujeres y femenina o femeninas \\
\hline Formas de nombrar & $\begin{array}{l}\text { Nombre de pila sin apellido, apellido de soltera y apellido de ca- } \\
\text { sada }\end{array}$ \\
\hline Verba dicendi & Verbos neutros o combativos \\
\hline \multicolumn{2}{|r|}{ Encuadre 4: Personalidad } \\
\hline Personalidad femenina & $\begin{array}{l}\text { Acciones o adjetivos que representen a la candidata como empáti- } \\
\text { ca, de bajo perfil, honesta, emocional, famosa o ambiciosa }\end{array}$ \\
\hline Personalidad masculina & $\begin{array}{l}\text { Acciones o adjetivos que representen a la candidata como lideresa, } \\
\text { políticamente fuerte, contestataria, dura, inteligente o deshonesta }\end{array}$ \\
\hline
\end{tabular}




\begin{tabular}{|l|l|}
\hline \multicolumn{2}{|c|}{ Encuadre 5: Agenda temática } \\
\hline Agenda femenina & $\begin{array}{l}\text { Agenda enfocada al ser para otros como ciencia y cultura, salud, } \\
\text { familia y sociedad, agenda feminista, educación, urbanismo y me- } \\
\text { dio ambiente. }\end{array}$ \\
\hline Agenda masculina & $\begin{array}{l}\text { Agenda enfocada a temas importantes para la defensa de la so- } \\
\text { beranía como economía, seguridad, corrupción, política exterior y } \\
\text { elecciones internas }\end{array}$ \\
\hline \multicolumn{1}{|l|}{ Encuadre 6: Tonalidad o valencia } \\
\hline Neutra & La candidata presenta sus propuestas sin sesgo evidente \\
\hline Positiva & $\begin{array}{l}\text { La sociedad civil apoya la candidatura y encuestas a favor de la } \\
\text { candidatura }\end{array}$ \\
\hline Negativa & $\begin{array}{l}\text { Grupos en contra de la candidatura, obtención de la candidatura } \\
\text { por medio de procedimientos poco claros, corrupción, conflicto de } \\
\text { interés, inviabilidad de la campaña, tono de los adjetivos, verbos } \\
\text { y adverbios }\end{array}$ \\
\hline \multicolumn{2}{|c|}{ Encuadre 7: Particularidades del medio } \\
\hline Sexo del periodista & Reportero, reportera y mixto \\
\hline Espectro político & De izquierda/liberal y derecha/conservador \\
\hline Tipología del medio & $\begin{array}{l}\text { Diario, tabloide, revista, página de internet, video, programa de te- } \\
\text { levisión o radio y podcast }\end{array}$ \\
\hline
\end{tabular}

Fuente: Elaboración propia.

El encuadre de mediatización de esfera íntima propone elaborar un análisis de todos los elementos que tienen que ver con apariencia física, tales como vestuario, peinado, posturas físicas, entre otros que indiquen que la cobertura tiene un distanciamiento de la información política para dedicarse a datos intrascendentes. Forman parte de este ámbito el conocer información familiar de la candidata como su estatus civil, número de hijos, datos de sus padres, hermanos y familia extensiva. Las mujeres generan incidencia en este espacio íntimo debido a que históricamente les han asignado labores del hogar, mientras que los hombres se encargan del espacio público.

El tercer encuadre hace referencia a la reiteración del género, el cual busca registrar la repetición sistemática de la palabra mujer, las maneras de nombrar a las candidatas, así como el tratamiento a través de verbos neutros o combativos. La idea es verificar qué tanta relevancia le dan los medios hacia el hecho de ser mujer, lo cual pondría una barrera en tanto que su tratamiento debería ser como una política. Los encuadres cuatro y cinco distinguen atributos y temas en masculinos y femeninos, de tal forma que son las del hombre la que más se acerca a una figura política ejemplar. 
El encuadre seis propone estudiar la valencia del mensaje periodístico ya que, aunque pueda existir una visibilidad alta y politización de los hechos de las candidatas, los medios podrían expresar a través de la tonalidad que las mujeres no son aptas para el puesto. Por último, el encuadre siete plantea estudiar las características del medio, en orden de poder comparar si la forma de tratamiento obedece al sexo del periodista, al giro del medio o al espectro político. Para esta última característica, se pueden utilizar los trabajos que buscan revelar la ideología de los medios de comunicación (Lawson, 2002; Ho \& Quinn, 2008; Rodelo \& Muñiz, 2017).

Dentro de estas mismas categorías es posible hacer varios cruces de variables. Uno de los más importantes es la agenda temática, la cual puede compararse con la visibilidad, por ejemplo, para saber en qué tipo de tópicos se utilizó la cita directa o se prefirió la paráfrasis. En ese sentido, se puede debatir con los autores Wasburn \& Wasburn (2011) si los redactores de las noticias prefieren darle voz a sus declaraciones cuando se tratan de temas de cuidado, mientras que parafrasean o no presentan los argumentos de las candidatas en temáticas de alto rango o masculinas.

Para comprobar si la valencia de las noticias es específica de un tipo de tema de personalidad, es posible cruzar el encuadre de tonalidad con el de personalidad y agenda temática. De esta manera, se pondrían a prueba los estudios que aseguran que la tonalidad neutral o positiva depende si las mujeres se presentan como femeninas (Harp, Loke \& Bachmann, 2010; Dan \& Iorgoveanu, 2013; Quevedo \& Suárez-Romero, 2017) y tocan temas sociales (Wachai, 2005).

Tabla 4: Indicadores de politización y despolitización.

\begin{tabular}{|l|l|l|}
\hline \multicolumn{1}{|c|}{ Indicador } & \multicolumn{1}{c|}{ Despolitización } & \multicolumn{1}{c|}{ Politización } \\
\hline Cantidad de cobertura & Baja & Alta o promedio \\
\hline Lenguaje de cobertura & Visual & Textual \\
\hline Género periodístico & Opinativos & Informativos \\
\hline Ubicación & $\begin{array}{l}\text { Páginas interiores o cuerpo del } \\
\text { programa }\end{array}$ & $\begin{array}{l}\text { Páginas principales o de por- } \\
\text { tada e inicio del programa }\end{array}$ \\
\hline Lugar en la noticia & En el cuerpo informativo & $\begin{array}{l}\text { Encabezado o promo de la } \\
\text { noticia }\end{array}$ \\
\hline Declaraciones & Paráfrasis o sin declaraciones & Citas directas o bites \\
\hline
\end{tabular}




\begin{tabular}{|l|l|l|}
\hline Personaje noticioso & $\begin{array}{l}\text { Personajes que acompañan a la } \\
\text { candidata }\end{array}$ & Ella misma \\
\hline Imagen física & $\begin{array}{l}\text { Cobertura hacia la apariencia } \\
\text { física }\end{array}$ & Sin cobertura \\
\hline Tipos de credenciales & Personales & Profesionales \\
\hline Reiteración del género & Aparición palabra mujer & Sin palabra mujer \\
\hline Formas de nombrarla & Nombre de pila sin apellido & Apellido de soltera o familiar \\
\hline Tipo de verbos & Emocionales o combativos & $\begin{array}{l}\text { Verbos neutros o combativos } \\
\text { en temas necesarios }\end{array}$ \\
\hline Tipo de personalidad & Femenina & Masculina \\
\hline Tipo de agenda & Femenina & Masculina \\
\hline Tonalidad & Negativa & Neutral o positiva \\
\hline
\end{tabular}

Fuente: Elaboración propia.

Un cruce más es el que tiene que ver con la manera en la que se nombra a la candidata presidencial de acuerdo con el tópico sobre el que genera postura. En ese sentido, Page (2003) sostiene que la elección de si se coloca el nombre o apellido obedece al tipo de temáticas que trata.

En la tabla 4, se encuentran todos los indicadores en dos clasificaciones: la despolitización y la politización. La primera se refiere a aquellos rasgos que le restan formalidad a la candidatura o imposibilitan una representación lo suficientemente política para evidenciar sus propuestas. La segunda fija ciertos estilos de tratamiento que permiten a las candidatas expresarse de una manera política y formal.

\section{A modo de cierre}

Los encuadres propuestos en este trabajo son concebidos con la finalidad de reparar si el tratamiento periodístico hacia candidatas presidenciales obedece a la estructura culturalmente creada sobre la posición de las mujeres en las esferas de poder, o si han generado nuevos paradigmas de cobertura. En ese sentido, encuadres como el de visibilidad, plantean que una mayor exposición, contemple una mayor consideración hacia los cargos de elección popular. De Botton (2001) explica que exponerse a la otredad y sus necesidades, permite su aceptación y la creación de una nueva cultura. 
Durante el apartado del escenario actual de las candidatas latinoamericanas se evidenció una baja participación de mujeres nominadas para el puesto ejecutivo, así como poca intención del voto. Anzaldúa (1987) explicó que los medios y la sociedad en general no concibe a las mujeres en los espacios de poder debido a que estos son creados por y para el hombre, lo que genera una posición de ventaja para este, puesto que el pueblo al que intenta gobernar se encuentra predispuesto a ver a los hombres en cargos de poder, mientras que las mujeres son las transmisoras de esa idea estructural de poder.

Los resultados de los estudios presentados durante los antecedentes apuntan a que existe una despolitización hacia las candidatas por parte de los medios, ya que son presentadas más que como políticas, como mujeres. Para Benhabib (1992), los medios deberían participar en la creación de narrativas que vuelvan porosa la frontera entre el espacio íntimo y el público, de tal manera que las mujeres puedan expresarse tanto en la esfera privada como pública.

Aunque pareciera que las mujeres ocupan cada vez más los lugares de poder, Valcárcel (1997) afirma que lo hacen a través de dos formas particulares. Primero, lo realizan sin la completa investidura, esto es, que el poder viene delegado, de alguna u otra forma, directamente de hombres. El segundo rasgo es que las mujeres detentan el poder con los valores religiosos de pobreza, castidad y obediencia, así como las virtudes griegas femeninas de la fidelidad y la abnegación. En ese sentido, las mujeres políticas no gozan de muchas posibilidades para moverse dentro de la política, puesto que su género parece darle una personalidad y agenda categóricas.

Por todo ello, resulta relevante reparar en la participación de los medios al abordar candidaturas femeninas, debido a que podrían abonar al fenómeno del techo de cristal, que se da la ilusión de que las condiciones de acceso de la mujer al poder están ahí, pero que ellas no quieren acceder a él.

En cuanto a su metodología, es relevante reparar en que la elaboración de encuadres se realizó de manera deductiva ya que se definieron previamente los encuadres de forma teórica (Igartua \& Humanes 2004). Sin embargo, su operacionalización no es cerrada, al contrario, su constante adaptación es deseable, en orden de revelarse nuevos marcos de referencia o desestimar algunos. Así, la propuesta se trata de que dichos encuadres funcionen como andamiaje teórico-metodológico desde el cual partir la investigación. 


\section{Fuentes consultadas}

Aalberg, T. \& Jenssen, A. (2007). Gender stereotyping of political candidates. An experimental study of political communication. Nordicom Review, 28(1), 17-32. doi: 0.1515/nor-2017-0198

Adams, K. (2011). The naked truth: the media's role in undermining female political candidates. International Journal of Politics and Good Governance, 2(2). Recuperado de http://onlineresearchjournals.com/ijopagg/ art/86.pdf

Aday, S. \& Devitt, J. (2001). Style over substance: newspaper coverage of Elizabeth Dole's Presidential Bid. The International Journal of Press/ Politics, 6(2), 52-73. doi: 10.1177/108118001129172134

Agostinho Martins, C. (2013). Mulheres, Política e Visibilidade Mediática. As Lideranças de Maria de Lourdes Pintasilgo e de Manuela Ferreira Leite (tesis doctoral). Universidade Nova de Lisboa, Portugal. Recuperado de https://bit.ly/32GZxbL

Allen, R. (2016). Gender, media, and the White House: an examination of gender in the media coverage of Hillary Clinton, Bernie Sanders, and Ted Cruz. Political Science Honors Projects, Paper 55. Recuperado de https://bit.ly/2OaATNf

Anderson, K. \& Sheeler, K. (2005). Governing Codes. Gender, Metaphor, and Political Identity. Nueva York: Lexington Books.

Anzaldúa, G. (1987). Borderlands/La Frontera: The New Mestiza. San Francisco: Aunt Lute Books.

Araujo, C. \& Alves, J. (2007). Impactos de indicadores sociais e do sistema eleitoral sobre as chances das mulheres nas eleições e suas interações com as cotas. Dados, 50(3), 535-577. doi: 10.1590/S001152582007000300004

Arendt, H. (2009). La condición humana. Buenos Aires: Paidós. 
Aruguete, N. (2011). Framing. La perspectiva de las noticias. La Trama de la Comunicación, 15(1), 67-80. Recuperado de https://latrama.fcpolit. unr.edu.ar/index.php/trama/article/view/52

Atkeson, L.\& Krebs, T. (2008). Press coverage of mayoral candidates: the role of gender in news reporting and campaign issue speech. Political Research Quarterly, 61(2), 239-252. doi:10.1177/1065912907308098

Banwart, M., Bystrom, D. \& Robertson, T. (2003). From the primary to the general election. A comparative analysis of candidate media coverage in mixed-gender 2000 races for governor and U.S. Senate. American Behavioral Scientist, 46(5), 658-676. doi: 10.1177/0002764202238491

Benhabib, S. (1992). Situating the Self: Gender, Community and Postmodernism in Contemporary Ethics. Nueva York: Routledge.

Berrocal, S. \& Quevedo, R. (2014). La representación de los políticos en revistas femeninas de alta gama. Un estudio de caso. Communication Papers, 3(5), 61-73. Recuperado de https://bit.ly/2McErvI

Bertrand, A. (2016). A Content Analysis of Media Coverage of Female U.S. Senate Candidates from the South (tesis de maestría). University of Louisiana, Lafayette, Estados Unidos.

Bijker, A. (2015). Gender Differences in the Dutch Media. Three Mixed-Gender Elections Investigated (tesis de maestría). Universiteit Leiden, Leiden, Países Bajos. Recuperado de https://bit.ly/2Yj5wUG

Bryant, L. (2014). Gender Balanced or Gender Biased? An Examination of News Coverage of Male and Female Governors (tesis doctoral). State University of New York, Albany, Estados Unidos.

Bystrom, D., Robertson, T., \& Banwart, M. (2001). Framing the fight. An analysis of media coverage of female and male candidates in primary races for governor and U.S. Senate in 2000. American Behavioral Scientist, 44(12), 1999-2013. doi: 10.1177/00027640121958456 
Carlin, D. \& Winfrey, K. (2009). Have you come a long way, baby? Hillary Clinton, Sarah Palin, and sexism in 2008 campaign coverage. Communication Studies, 60(4), 326-343. doi: 10.1080/10510970903109904

Cassidy, K. (2012). Bringing the Frame into Focus: How Cable News Pundits Protect the Glass Ceiling (tesis de maestría). University of Massachusetts, Amherst, Estados Unidos.

Clark, K. (1992). The linguistics of blame: representations of women in The Sun's reporting of crimes of sexual violence. En M. Toolan (Ed.), Language, Text and Context: Essays in Contextualised Stylistics (pp. 208-224). Londres: Routledge.

Conroy, M., Oliver, S., Breckenridge-Jackson, I. \& Heldman, C. (2015). From Ferraro to Palin: sexism in coverage of vice presidential candidates in old and new media. Politics, Groups, and Identities, 3(4), 573-591. doi: 10.1080/21565503.2015.1050412

Costa, L., Bolognesi, B. \& Codato, A. (2013). Variáveis sobre o recrutamento político e a questão de gênero no Parlamento brasileiro. The Observatory of Social and Political Elites of Brazil, 7, 1-20. Recuperado de http://observatory-elites.org/wp-content/uploads/2012/06/wp-observatory-n.7-2013.pdf

Dan, V. \& Iorgoveanu, A. (2013). Still on the beater path. How gender impacted the coverage of male and female Romanian candidates for European office. The International Journal of Press/Politics, 18(2), 208233. doi: $10.1177 / 1940161212473508$

De Botton, A. (2001). Sobre la ineptitud cultural. En A. de Botton (Ed.), Las consolidaciones de la filosofía (pp. 145-163). México, DF: Taurus.

De Fontcuberta, M. (1990). El discurso de la prensa del corazón. Anàlisi, 13(1), 53-72. Recuperado de https:/www.raco.cat/index.php/analisi/ article/viewFile/41093/89101

De Vreese, C. \& Boomgaarden, H. (2003). Valenced news frames and public support for the EU. Communications, 28(4), 361-381. doi: 10.1515/ comm.2003.024 
Ditonto, T. (2014). The outsider and the presidency. Mediated representations of race and gender in the 2008 presidential primaries. En G. Daniel \& H. Williams (Eds.), Race and the Obama Phenomenon: The Vision of a More Perfect Multiracial Union. Mississippi: University Press of Mississippi. doi: 10.14325/mississippi/9781628460216.003.0010

Dunaway, J., Lawrence, R., Rose, M. \& Weber, C. (2013). Traits versus issues: how female candidates shape coverage of Senate and gubernatorial races. Political Research Quarterly, 66(3), 715-726. doi: $10.1177 / 1065912913491464$

Ehrlich, S. \& King, R. (1992). Gender-based language reform and the social construction of meaning. Discourse \& Society, 3(2), 151-166. doi: 10.1177/0957926592003002002

Entman, R. (1993). Framing: Toward clarification of a fractured paradigm. Journal of Communication, 43(4), 51-58. doi: 10.1111/j.14602466.1993.tb01304.x

Everitt, J. (2003). Media in the maritimes: do female candidates face a bias? Atlantis, 27(2), 90-98. Recuperado de http://140.230.24.104/index. php/atlantis/article/view/1325/1197

Falk, E. (2010). Women for President: Media Bias in Nine Campaigns. Champaign: University of Illinois Press.

Fernández García, N. (2010). Framing Hillary Clinton in Spanish Print Media: Candidate or Woman? Observatorio Journal, 4(3), 209-228. Recuperado de https://core.ac.uk/download/pdf/18417596.pdf

Fernández García, N. \& Tous Rovirosa, A. (2012). La representación de las mujeres políticas en los medios. En A. Larrondo \& K. Meso (Eds.), Mujer y Medios de Comunicación, 4, 23-44). Barcelona: Universidad del País Vasco.

Fernández Poncela, A. (2008). Las mujeres en la política latinoamericana. Nuevos liderazgos, viejos obstáculos. Nueva Sociedad, (218), 57-71. Recuperado de https://nuso.org/articulo/nuevos-liderazgos-viejos-obstaculos 
Finamore, C. \& Coin de Carvalho, J. (2006). Mulheres candidatas: relações entre gênero, mídia e discurso. Estudos Feministas, 14(2), 347-362. doi: 10.1590/S0104-026X2006000200002

Foster, N. \& Parsons, T. (2016). 18 million cracks, but no cigar: news media and the campaigns of Clinton, Palin, and Bachmann. Social Sciences, 5(3). doi: 10.3390/socsci5030050

Franceschet, S. (2006). El triunfo de Bachelet y el ascenso político de las mujeres. Nueva Sociedad, 202, 13-22. Recuperado de https://nuso.org/ articulo/el-triunfo-de-bachelet-y-el-ascenso-politico-de-las-mujeres/

Gamson, W. \& Modigliani, A. (1989). Media discourse and public opinion on nuclear power: a constructionist approach. The American Journal of Sociology, 95(1), 1-37. doi: 10.1086/229213

García Beaudoux, V. (2014). Influencia de la televisión en la creación de estereotipos de género y en la percepción social del liderazgo femenino. La importancia de la táctica de reencuadre para el cambio social. Ciencia Política, 9(18), 47-66. Recuperado de https://revistas.unal. edu.co/index.php/cienciapol/article/view/52305

García Beaudoux, V., D’Adamo, O. \& Gavensky, M. (2018). Una tipología de los sesgos y estereotipos de género en la cobertura periodística de las mujeres candidatas. Revista Mexicana de Opinión Pública, 13(24), 113-129. doi: 10.22201/fcpys.24484911e.2018.24.61614

García, A., Gómez-Escalonilla, G., Torregrosa, J., Rodríguez, R. \& Santín, M. (2008). Medios de comunicación, representaciones y percepciones. El caso de la mujer política. Estudios sobre el Mensaje Periodístico, 14, 175-196. Recuperado de https://bit.ly/2LASVWS

Gerbner, G. \& Gross, L. (1976). Living with television: the violence profile. Journal of Communication, 26(2), 172-194. doi: 10.1111/j.1460-2466.1976.tb01397.x

Gidengil, E. \& Everitt, J. (2003). Talking tough: gender and reported speech in campaign news coverage. Political Communication, 20(3), 209232. doi: 10.1080/10584600390218869 
Gómez-Escalonilla, G., García, A., Santín, M., Rodríguez, R. \& Torregrosa, J. (2008). La imagen de la mujer política en los medios de comunicación. Feminismo/s, 11, 59-71. doi: 10.14198/fem.2008.11.04

Habermas, J. (1989). The Structural Transformation of the Public Sphere. Cambridge: The MIT Press.

Hall, S. (1974). Media power: the double bind. Journal of Communication, 24(4), 19-26. doi: 10.1111/j.1460-2466.1974.tb00404.x

Harp, D., Loke, J. \& Bachmann, I. (2010). First impressions of Sarah Palin: pit bulls, politics, gender performance, and a discursive media (re) contextualization. Communication, Culture \& Critique, 3(3), 291309. doi: 10.1111/j.1753-9137.2010.01072.x

Heldman, C., Carroll, S. \& Olson, S. (2005). "She brought only a skirt": print media coverage of Elizabeth Dole's bid for the Republican Presidential nomination. Political Communication, 22(3), 315-335. doi: $10.1080 / 10584600591006564$

Ho, D. \& Quinn, K. (2008). Measuring explicit political positions of media. Quarterly Journal of Political Science, 3(4), 353-377. doi: $10.1561 / 100.00008048$

Holtz-Bacha, C. (2013). Who takes care of the children? The presentation of female top politicians in the media. Compolitica, 3(2), 45-76. Recuperado de https://bit.ly/2GpcPjw

Hooghe, M., Jacobs, L. \& Claes, E. (2015). Enduring gender bias in reporting on political elite positions. Media coverage of female MPs in Belgian news broadcasts (2003-2011). The International Journal of Press/Politics, 20(4), 395-414. doi: 10.1177/1940161215596730

Humprecht, E. \& Esser, F. (2017). A glass ceiling in the online age? Explaining the underrepresentation of women in online political news. European Journal of Communication, 32(5), 439-456. doi: $10.1177 / 0267323117720343$ 
Igartua, J. \& Humanes, M. (2004). Teoría e investigación en comunicación social. Madrid: Síntesis.

Jalalzai, F. (2006). Women candidates and the media: 1992-2000 elections. Politics \& Policy, 34(3), 606-633. doi: 10.1111/j.17471346.2006.00030.x

Jamieson, K. (1995). Beyond the Double Bind. Women and Leadership. Nueva York: Oxford University Press.

Kahn, K. (1992). Does being male help? An investigation of the effects of candidate gender and campaign coverage on evaluations of U.S. Senate candidates. The Journal of Politics, 54(2), 497-517. doi: $10.2307 / 2132036$

Kahn, K. (1994). The distorted mirror: press coverage of women candidates for statewide office. The Journal of Politics, 56(1), 154-173. doi: $10.2307 / 2132350$

Kahn, K. \& Goldenberg, E. (1991). Women candidates in the news: an examination of gender differences in U.S. Senate campaign coverage. The Public Opinion Quarterly, 55(2), 180-199. doi: 10.1086/269251

Kittilson, M. \& Fridkin, K. (2008). Gender, candidate portrayals and election campaigns: a comparative perspective. Politics \& Gender, 4(3), 371392. doi: $10.1017 / \mathrm{S} 1743923 \mathrm{X} 08000330$

Lawson, C. (2002). Building the Fourth Estate. Democratization and the Rise of a Free Press in Mexico. Los Ángeles: University of California Press.

Lemarier-Saulnier, C. \& Lalancette, M. (2012). La dame de fer, la bonne mère et les autres: une analyse du cadrage de la couverture médiatique de certaines politiciennes québécoises et canadiennes. Canadian Journal of Communication, 37, 459-486. doi: 10.22230/cjc.2012v37n3a2583

Llanos, B. (2014). Ojos que (Aún) No Ven. Nuevo reporte de ocho países: género, campañas electorales y medios en América Latina. Nueva York: IDEA, ONU. Recuperado de https://bit.ly/2OwzvB1 
López, P. \& Vicente, M. (2013). Propuestas de consolidación teórica y analítica para los estudios de framing en la investigación sobre comunicación política. En K. Sanders, M. Canel, A. Capdevila, \& M. Gurrionero (Eds.), Estudios de Comunicación Política (pp. 239-259). Madrid: Tecnos.

López-Hermida, A. (2009). La imagen de la mujer en política: la campaña electoral televisiva de Michelle Bachelet. Cuadernos.info, 24, 5-16. doi: $10.7764 /$ cdi.24.30

Lünenborg, M. \& Maier, T. (2014). "Power politician" or "Fighting bureaucrat": gender and power in German political coverage. Media, Culture \& Society, 37(2), 180-196. doi: 10.1177/0163443714557979

Maldonado, C. (1999). Discurso directo y discurso indirecto. En I. Bosque \& V. Demonte (Eds.), Gramática Descriptiva de la Lengua Española (pp. 3549-3596). Madrid: Espasa.

Martínez, F. \& Godínez, F. (2013). La agenda de los telediarios en la contienda del 2012. Derecho a Comunicar, 7(1), 59-75. Recuperado de https://bit.ly/2LE2ggG

Martínez, F. \& Maltos, A. (2019). La elección federal en telediarios públicos. Revista Mexicana de Opinión Pública, 14(27), 79-93. doi: 10.22201/ fcpys.24484911e.2019.27.68549

Martínez-Lirola, M. (2010). Explorando la invisibilidad de mujeres de diferentes culturas en la sociedad y en los medios de comunicación. Palabra Clave, 13(1), 161-173. doi: 10.5294/pacla.2010.13.1.10

Meeks, L. (2013). All the gender that's fit to print. How the New York Times covered Hillary Clinton and Sarah Palin in 2008. Journalism \& Mass Communication Quarterly, 90(3), 520-539. doi: $10.1177 / 1077699013493791$

Miller, M. \& Peake, J. (2013). Press effects, public opinion, and gender: coverage of Sarah Palin's vice-presidential campaign. The International Journal of Press/Politics, 18(4), 482-507. doi: $10.1177 / 1940161213495456$ 
Monfardini, M. (2013). O Feminino nas Eleições de 2010: O Discurso de Veja e Isto sobre as Mulheres Candidatas (Pós-Graduacao em Teoria do Jornalismo e Comunicação). Universidade de Brasília, Brasil.

Morales, M. (2008). La primera mujer presidenta de Chile: ¿qué explicó el triunfo de Michelle Bachelet en las elecciones de 2005-2006? Latin American Research Review, 43(1), 7-32. Recuperado de https://www. jstor.org/stable/20488107?seq=1\#page_scan_tab_contents

Page, R. (2003). “Cherie: lawyer, wife, mum”: contradictory patterns of representation in media reports of Cherie Booth/Blair. Discourse \& Society, 14(5), 559-579. doi: 10.1177/09579265030145002

Power, K. (2017). Church trailblazer Rev Pat Storey on weight watchers, caffeine and how she named her dog after former New York Mayor: news representations of the first female Anglican Bishop in the UK and Ireland. Irish Journal of Applied Social Studies, 16(1). doi: 10.21427/D78T67

Quevedo, R. \& Suárez-Romero, M. (2017). Del retrato de la Dama de Hierro a la fotografía de Mutti Angela: la representación icónica de la canci1ler alemana Angela Merkel. Estudios sobre el Mensaje Periodístico, 23(1), 535-551. doi: 10.5209/ESMP.55612

Rincón Soto, L. (2015). Mujer y poder político en Latinoamérica: Una reflexión desde el siglo XXI. Utopía y Praxis Latinoamericana, 20(68), 65-78. Recuperado de http://www.redalyc.org/articulo. oa? id=27937090006

Ríos Sierra, J. (2017). Liderazgo político y patriarcado mediático: las imágenes políticas de Cristina Fernández y Dilma Rousseff. Revista de Investigaciones Políticas y Sociológicas, 16(1), 65-86. doi: 10.15304/ rips.16.1.3461

Rodelo, F. (2016). Disparidades de género en la cobertura mediática de campañas electorales locales en México. Cuadernos.info, 39, 87-99. doi: 10.7764/cdi.39.965 
Rodelo, F. \& Muñiz, C. (2017). La orientación política del periódico y su influencia en la presencia de encuadres y asuntos dentro de las noticias. Estudios sobre el Mensaje Periodístico, 23(1), 241-256. doi: 10.5209/ ESMP.55594

Ross, K. (2002). Women, Politics, Media: Uneasy Relation in Comparative Perspective. Cresskill: Hampton Press.

Sánchez Ruiz, E. (2005). Medios de comunicación y democracia. Bogotá: Norma.

Schuck, A. \& De Vreese, C. (2006). Between risk and opportunity: News framing and its effects on public support for EU enlargement. Journal of Communication, 21(1), 5-32. doi: 10.1177/0267323106060987

Semetko, H. \& Boomgaarden, H. (2007). Reporting Germany's 2005 Bundestag election campaign: was gender an issue? The International Journal of Press/Politics, 12(4), 154-171. doi: 10.1177/1081180X07307383

Semetko, H. \& Valkenburg, P. (2000). Framing european politics: A content analysis of press and television news. Journal of Communication, 50(2), 93-109. doi: 10.1111/j.1460-2466.2000.tb02843.x

Sensales, G., Areni, A. \& Dal Secco, A. (2016). Linguistic sexism in the news coverage of women ministers from four Italian governments. Journal of Language and Social Psychology, 35(4), 458-466. doi: 10.1177/0261927X16629787

Stein, K. (2009). The cleavage commotion: how the press covered senator Clinton's campaign. En T. Sheckels (Ed.), Cracked but no Shattered: Hillary Rodham Clinton's Unsuccessful Campaign for the Presidency (pp. 173-188). Lanham: Lexington Books.

Tankard, J. (2001). The empirical approach to the study of media framing. En S. Reese, O. Gandy \& A. Grant (Eds.), Framing Public Life. Perspectives on Media and our Understanding of the Social World (pp. 95-106). Nueva Jersey: Lawrence Erlbaum Associates. 
Trimble, L., Wagner, A., Sampert, S., Raphael, D., \& Gerrits, B. (2013). Is it personal? Gendered mediation in newspaper coverage of Canadian National Party leadership contests, 1975-2012. The International Journal of Press/Politics, 18(4), 462-481. doi: 10.1177/1940161213495455

Uscinski, J. \& Goren, L. (2010). What's in a Name? Coverage of Senator Hillary Clinton during the 2008 Democratic Primary. Political Research Quarterly, 64(4), 884-896. doi: 10.1177/1065912910382302

Valcárcel, A. (1997). La politica de las mujeres. Madrid: Cátedra.

Valcárcel, A. (2008). Feminismo en el mundo global. Madrid: Cátedra.

Valenzuela, S. \& Correa, T. (2006). Prensa y candidatos presidenciales 2005. Así los mostramos, así los miraron. Cuadernos de Información, 19, 89-96. doi: 10.7764/cdi.19.129

Van Acker, E. (2003). Media representations of women politicians in Australia and New Zealand: high expectations hostility or stardom. Policy and Society, 22(1), 116-136. doi: 10.1016/S14494035(03)70016-2

VanHorn, A. (2010). Candidate Wives: Spouses as Strategic Surrogates on the Presidential Campaign Trail (tesis doctoral). Lafayette: Purdue University.

Vicente Mateu, J. A. (2007). Discurso reproducido e interpretación de la fuerza ilocutiva en la prensa escrita. Revista de Investigación Lingüistica, (10), 225-242. Recuperado de https://revistas.um.es/ril/article/ view/4831

Vos, D. (2013). The vertical glass ceiling: explaining female politicians' underrepresentation in television news. Communications, 38(4), 389410. doi: 10.1515/commun-2013-0023

Wachai, J. (2005). Journalistic Gender Stereotyping of First Ladies Laura Bush and Hillary Clinton (tesis de maestría). Edgerton University, Kenia. 
Wagner, A., Trimble, L., Sampert, S., \& Gerrits, B. (2017). Gender, competitiveness and candidate visibility in newspaper coverage of Canadian party leadership contests. The International Journal of Press/Politics, 22(4), 471-489. doi: 10.1177/1940161217723150

Wasburn, P. \& Wasburn, M. (2011). Media coverage of women in politics: The curious case of Sarah Palin. Media Culture \& Society, 33(7), 1027-1041. doi: 10.1177/0163443711415744

Whalen, S. (2016). Coverage of the 2008 Presidential primary campaign by male, females, and mixed journalist groups. Speaker \& Gavel, 53(1). Recuperado de https://cornerstone.lib.mnsu.edu/speaker-gavel/vol53/iss1/9

White, S. (2012). Mothers and whores: the relationship between popular culture and women in politics. Mapping Politics, 4(1). Recuperado de https:// journals.library.mun.ca/ojs/index.php/MP/article/view/370/242 